\title{
Variações na riqueza e na diversidade de espécies arbustivas e arbóreas no período de 14 anos em uma Floresta de Vale, Mato Grosso, Brasil
}

Variations in richness and tree species diversity within 14 years in a valley forest, Mato Grosso, Brazil

\author{
Thiago Ayres Lazzarotti Abreu ${ }^{1,3}$, José Roberto Rodrigues Pinto $^{2}$ \& Henrique Augusto Mews ${ }^{1}$
}

\begin{abstract}
Resumo
O presente trabalho teve como objetivo descrever variações na riqueza, na diversidade e na contribuição dos grupos ecológicos para a composição florística da vegetação arbustiva e arbórea (DAP $\geq 5 \mathrm{~cm}$ ) na Floresta de Vale no Véu de Noiva (FVVN), localizada no Parque Nacional da Chapada dos Guimarães, Mato Grosso, Brasil, num período de 14 anos, com base em cinco inventários (1996, 1999, 2003, 2006 e 2010). Todas as espécies arbustivas e arbóreas (DAP $\geq 5 \mathrm{~cm}$ ) foram amostradas sistematicamente em 18 parcelas de $600 \mathrm{~m}^{2}$ distribuídas em três transecções no vale. As mudanças na riqueza de espécies resultaram em aumento, porém não significativos, nos intervalos menores (1996-1999, 1999-2003, 2003-2006 e 2006-2010) enquanto no intervalo maior (1996 a 2010) o aumento foi significativo (comparações entre contagens de Poisson, $p<0,05$ ) e caracterizado por acréscimo líquido de 20 espécies. Apesar disso, a manutenção da equabilidade determinou ausência de alterações significativas na diversidade de espécies ao longo do período estudado (teste $t$ de Hutcheson, $p>0,05$ ). A contribuição dos grupos ecológicos para a composição florística também não apresentou mudanças significativamente durante os 14 anos de monitoramento. Estes resultados sugerem uma comunidade com dinâmica acelerada na composição florística, porém com manutenção temporal da diversidade e da distribuição das espécies entre os grupos ecológicos. Palavras-chave: comunidades, dinâmica, Floresta Estacional Semidecidual, Floresta de Galeria, Parque Nacional da Chapada dos Guimarães.
\end{abstract}

\begin{abstract}
The goal of this study was evaluate changes in richness, diversity and the contribution of successional groups to tree species composition of a Valley Forest in the Chapada dos Guimarães National Park, Mato Grosso State, Brazil, over 14 years (1996 - 2010) from five surveys intervals (1996-1999, 1999-2003, 2003-2006, 2006-2010, 1996-2010). All tree species $(\mathrm{dbh} \geq 5 \mathrm{~cm})$ were sampled systematically in a total of 18 plots $\left(600 \mathrm{~m}^{2}\right)$ established into three transects in valley. Whereas the changes in species richness were positive, but barely noticeable when analyzed in shorter intervals, the range considering the whole interval (1996-2010) was significant (comparisons of Poisson counts) with a net increase of 20 species. Despite the observed species turnover, maintaining evenness seems to have given the absence of significant changes in diversity over the period studied (Hutcheson $t$ test). The contribution of successional groups to the tree species composition of tree community also did not show significant changes during 14 years of monitoring. These results suggest that tree community dynamics of a Valley forest is accelerated in tree species composition, although maintaining temporal diversity and distribution of species among successional groups. Key words: communities, dynamic, Semideciduous forest, Gallery Forest, Chapada dos Guimarães National Park.
\end{abstract}

\section{Introdução}

Várias teorias tentaram explicar os padrões de diversidade em florestas tropicais por meio da dicotomia entre ecossistemas em equilíbrio e nãoequilíbrio (Connell 1978; Terborgh 1992). Nestas teorias, geralmente é destacado o papel da dinâmica florestal como mecanismo regulador da situação de equilíbrio (Felfili 1995a). As interações temporais e espaciais dos processos demográficos da vegetação, tais como mortalidade, recrutamento e crescimento, são cruciais na determinação de nichos para o estabelecimento e a manutenção das espécies nas comunidades (Swaine et al. 1987; Swaine 1990; Condit 1995; Rees et al. 2001). São estas interações

\footnotetext{
${ }^{1}$ Universidade de Brasília - UnB, Programa de Pós-graduação em Ciências Florestais, C.P. 4357, 70919-970, Brasília, DF, Brasil.

${ }^{2}$ UnB, Depto. Engenharia Florestal, C.P. 4357, 70919-970, Brasília, DF, Brasil.

${ }^{3}$ Autor para correspondência: thiagoayresflorestal@gmail.br
} 
que determinam as principais características das comunidades florestais, como composição e diversidade de espécies (Tilman et al. 1997), além da sua estrutura (Oliveira-Filho et al. 1997). Nesse sentido, estudos de dinâmica da vegetação são de extrema importância para o entendimento de diversos processos ecológicos, entre os quais estão inclusos os padrões e a manutenção da riqueza de espécies (Phillips \& Sheil 1997; Baker et al. 2003) e a caracterização e compreensão da diversidade e complexidade estrutural das comunidades vegetais (Rees et al. 2001).

Em florestas tropicais isentas de distúrbios severos, geralmente são registradas poucas mudanças na densidade e na composição de espécies, o que sugere condição de aparente equilíbrio (Swaine et al. 1987; Laurance et al. 2006), também denominado de equilíbrio dinâmico (Felfili 1995b). Por outro lado, transformações na composição e estrutura são relatadas para florestas atingidas por grandes distúrbios ou interferências antrópicas (Laurance et al. 2004; Phillips et al. 2010). No Brasil, mesmo que sob diferentes metodologias e abordagens, estudos de dinâmica florestal na região extra-amazônica, realizados em florestas de galeria ou ciliares (Felfili 1995a,b; Bertani et al. 2001; Schiavini et al. 2001; Pinto \& Hay 2005; Lopes \& Schiavini 2007; Guimarães et al. 2008; Miguel et al. 2011) e em florestas estacionais (Oliveira-Filho et al. 1997; Aquino et al. 1999; Schiavini et al. 2001; Paiva et al. 2007; Paula et al. 2004; Appolinário et al. 2005; Oliveira-Filho et al. 2007; Higuchi et al. 2008; Machado \& Oliveira-Filho 2010; Carvalho \& Felfili 2011; Mews et al. 2012) têm demonstrado dinâmica acelerada para o componente arbustivo e arbóreo, embora isso não indique, necessariamente, grandes mudanças na composição e na estrutura da vegetação devido às reposições.

Apesar disso, é importante destacar que a maioria dos trabalhos realizados fora da região Amazônica analisou a dinâmica a partir de intervalo definido por duas mensurações, o que, de certa forma, limita a interpretação dos resultados. Nesse contexto, o presente trabalho tem por objetivo avaliar as mudanças na riqueza, na diversidade e na contribuição dos grupos ecológicos para a composição florística da vegetação arbustiva e arbórea da Floresta de Vale do Véu de Noiva (FVVN) no período de 14 anos (1996 a 2010), com base em cinco inventários consecutivos. Para tanto, procurou-se responder às seguintes questões: a riqueza e a diversidade de espécies arbustivas e arbóreas observadas na FVVN mudaram ao longo do período avaliado? O número de espécies arbustivas e arbóreas nos grupos ecológicos mudou ao longo do período avaliado?

\section{Material e Métodos}

Área de estudo

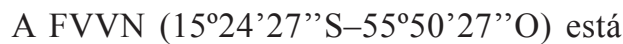
localizada no Parque Nacional da Chapada dos Guimarães (PNCG), na porção centro-sul do estado do Mato Grosso, Brasil (Fig. 1) e se encontra encaixada no vale situado logo após a queda d'água Véu de Noiva, formada pelo Córrego Coxipozinho. O vale está situado em uma depressão de aproximadamente $70 \mathrm{~m}$ em relação às áreas adjacentes e ocupa aproximadamente 30 ha, os quais representam a maior área florestal contínua do Parque (Pinto \& Oliveira-Filho 1999). Devido à difícil separação entre a vegetação da Floresta de Galeria e da Floresta de Encosta, denominadas, respectivamente, de Floresta Estacional Semidecidual Aluvial e Floresta Estacional Semidecidual (IBGE 2012), a formação florestal estudada é mais bem definida como 'Floresta de Vale' (sensu Eiten 1994), conforme adotado por Pinto \& Oliveira-Filho (1999), Pinto et al. (2005) e Pinto \& Hay (2005).

As condições climáticas do PNCG apresentam caráter transicional devido principalmente às diferenças de altitude entre as regiões da Depressão Cuiabana (350 m) e do Planalto $(800 \mathrm{~m})$, as quais possuem clima classificado, respectivamente, como Aw e Cw, segundo Köppen (ICMBio 2009). Ambos

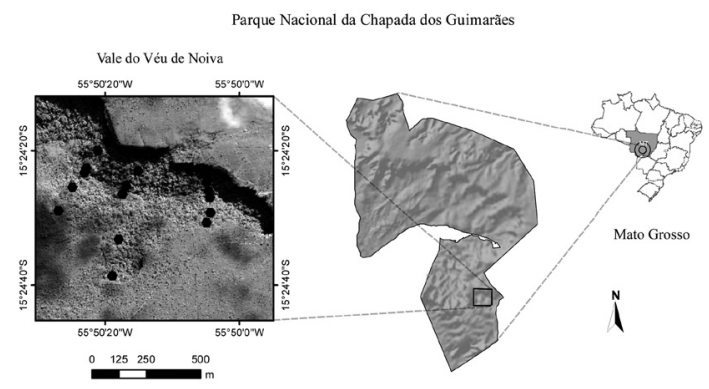

Figura 1 - Localização do Parque Nacional da Chapada dos Guimarães em relação ao Brasil e ao Estado de Mato Grosso, com destaque para a disposição das parcelas na área de estudo.

Figure 1 - Chapada dos Guimarães National Park localization with emphasis on the provision of plots in the study area. 
se caracterizam por serem quentes e úmidos, com duas estações bem definidas, sendo uma chuvosa de outubro até março (primavera e verão) e outra seca entre abril e setembro (outono e inverno). A temperatura normalmente varia de $12^{\circ} \mathrm{C}$ a $25^{\circ} \mathrm{C}$ e o total pluviométrico anual situa-se entre $1.650 \mathrm{~mm}$ e $2.100 \mathrm{~mm}$ (Ibama 1994).

De acordo com Pinto \& Oliveira-Filho (1999), no vale do Véu de Noiva predominam os solos Litólicos fase arenosa, mas manchas de areia quartzosa distrófica e solos aluviais também podem ocorrer. Esses autores comentam ainda que, em geral, o solo é raso e com constantes afloramentos rochosos, provavelmente em função do relevo acidentado.

\section{Inventários da vegetação}

Os inventários da vegetação seguiram o desenho amostral adotado por Pinto \& OliveiraFilho (1999). O estudo foi conduzido em três transeções paralelas distribuídas sistematicamente em diferentes seções do vale: próximo à queda d'água, no meio do vale e na extremidade oposta à queda d'água. Cada transeção foi formada por seis pontos de amostragens instalados em três setores topográficos: beira do córrego, meio e alto da encosta, em ambas vertentes do vale (Pinto \& Oliveira-Filho 1999; Pinto \& Hay 2005; Pinto et al. 2005; Fig. 1). Em cada ponto foram instaladas sistematicamente parcelas de $600 \mathrm{~m}^{2}$ cuja forma variou de acordo com o setor topográfico. No meio e no alto da encosta as parcelas foram de $20 \times 30 \mathrm{~m}$ e na beira do córrego as parcelas foram de $10 \times 60$ m (Pinto \& Oliveira-Filho 1999; Pinto et al. 2005). O formato das parcelas da beira do córrego diferiu das demais no intuito de maximizar a representação da comunidade influenciada pelo curso d'água (Pinto \& Oliveira-Filho 1999).

No inventário realizado em 1996 foram plaqueados e amostrados todos os indivíduos arbustivos e arbóreos com diâmetro à altura do peito (DAP) ou média quadrática das ramificações $\geq 5 \mathrm{~cm}$. Os indivíduos amostrados tiveram os valores do DAP, altura total, identificação taxonômica e hábito registrados. Nos inventários posteriores (1999, 2003, 2006 e 2010) foram avaliados os indivíduos sobreviventes e os recrutas (aqueles que atingiram o critério de inclusão). Não foram amostrados indivíduos mortos nem lianas, conforme método adotado por Pinto \& OliveiraFilho (1999) e Pinto \& Hay (2005). Cabe ressaltar que em 2010, três meses antes da realização do último inventário, a vertente norte do vale foi atingida por fogo.

As espécies registradas pela primeira vez nos inventários tiveram material botânico coletado, com testemunho depositado nos Herbários da Universidade Federal de Mato Grosso (HCUFMT) e da Universidade de Brasília (UB). Os indivíduos não identificados no campo foram coletados e herborizados, para posterior identificação. A identificação botânica das espécies foi realizada por meio de consulta à literatura específica, aos especialistas e por comparações com os acervos dos Herbários HCUFMT e UB. A revisão e a atualização dos nomes dos táxons foram realizadas no banco de dados da Lista de Espécies Flora do Brasil (Forzza et al. 2012).

\section{Análise de dados}

Os dados da comunidade foram analisados para cinco intervalos, definidos com base nos cinco inventários realizados. O intervalo compreendido entre os anos de 1996 e 2010 foi denominado intervalo $\mathrm{TG}$ e os intervalos menores foram denominados T1 (1996 a 1999), T2 (1999 a 2003), T3 (2003 a 2006), T4 (2006 a 2010).

A riqueza de espécies foi registrada para cada inventário e também foram calculados os estimadores "Jackknife" de primeira ordem. Este estimador projeta a riqueza de espécies da comunidade a partir do número de espécies registradas em apenas uma unidade amostral (uniques), ou seja, a partir da heterogeneidade da amostra (Magurran 2004). Com o propósito de detectar possíveis alterações na riqueza, ganhos e perdas de espécies foram contabilizados e contrastados em cada intervalo por meio de comparações entre contagens de Poisson (Zar 1999). Além disso, as riquezas do primeiro (1996) e do último inventários (2010) foram comparadas por meio de curvas de acumulação de espécies geradas por rarefação, seguindo a fórmula analítica de "Mao Tau" (Colwell et al. 2004), com auxílio do programa EstimateS 8.2.0 (Colwell 2006). A comparação entre essas duas curvas foi realizada graficamente a partir dos intervalos de confiança de $95 \%$ (Zar 1999).

De forma complementar, com base no método de rarefação, foi realizada comparação da riqueza entre os inventários de 1996 e 2010, empregandose o programa EcoSim 7.0 (Gotelli \& Entsminger 2001). Esta análise permite fixar na amostra número de indivíduos menor do que o realmente 
observado, para efeito de ajuste do esforço amostral em comparações. Assim, a riqueza foi estimada para o inventário de 2010 com a mesma densidade de indivíduos observada no inventário de 1996. A partir da média de espécies e variância fornecida pelo programa foi calculado o valor de " $p$ ", com base no teste $Z$ de distribuição normal padronizada (Zar 1999), para avaliar a significância da diferença entre as riquezas dos dois inventários (1996 e 2010).

A diversidade de espécies foi determinada, para cada ano inventariado, por meio do índice de Shannon (H', na base neperiana) (Magurran 2004) e a equabilidade pelo índice de Pielou (J') (Ludwing \& Reynolds 1988). Os valores de H' foram comparados entre todos os intervalos (T1, T2, T3, T4, TG) aplicando o teste $t$ de Hutcheson (Zar 1999). O índice de Shannon e os testes $t$ de Hutcheson foram calculados no programa PAST 2.08 (Hammer et al. 2001).

De forma adicional, para avaliar e comparar a diversidade de espécies entre os inventários, também foram empregados perfis de diversidade (Tóthmérész 1995), conforme recomendado por Melo (2008). De acordo com este autor, os perfis de diversidade são muito úteis para análise e comparação da diversidade de espécies, pois generalizam o peso que os diferentes índices podem dar às espécies raras (neste caso, pouco abundantes), evitando a escolha de um dado índice em detrimento de outro.

No presente estudo, os perfis de diversidade foram calculados a partir da série de Rényi, dada pela fórmula $\mathrm{H}_{\alpha}=\left(\ln \left(\mathrm{p}_{1}{ }^{\alpha}+\mathrm{p}_{2}{ }^{\alpha}+\mathrm{p}_{3}{ }^{\alpha} \ldots+\mathrm{P}_{\mathrm{s}}{ }^{\alpha}\right)\right) /(1-\alpha)$ com o auxílio do programa PAST 2.15 (Hammer et al. 2001). $H_{\alpha}$ é o valor do índice de diversidade para o parâmetro $\alpha(\alpha \geq 0, \alpha \neq 1)$ e $p 1, p 2, p 3 \ldots$ e $p n$ são proporções de indivíduos das espécies 1 , $2,3 \ldots S$. Melo (2008) indicou que quanto mais próximo de 0 for o fator $\alpha$, maior será o peso dado às espécies raras no índice $\mathrm{H}_{\alpha}$, de modo que quando $\alpha=0$ o valor do índice retornado é igual a riqueza de espécies, situação na qual é dado peso máximo às espécies raras.

As espécies foram classificadas em grupos ecológicos de acordo com a proposta de Swaine \& Whitmore (1988), modificada por Oliveira-Filho et al. (1994) e utilizada por Pinto \& OliveiraFilho (1999) no inventário realizado em 1996. Este sistema de classificação propõe a separação das espécies em três grupos ecológicos de acordo com sua estratégia de vida e suas exigências de luminosidade, sendo eles: clímax tolerante à sombra (CS), clímax exigente de luz (CL) e pioneiras (P). A determinação do grupo ecológico para cada espécie foi baseada nas características apresentadas pelos indivíduos jovens (imaturos) e adultos (fase reprodutiva), na consulta à literatura específica e nas informações disponíveis em várias listagens florísticas (Pinto \& Oliveira-Filho, 1999). As alterações na distribuição das espécies entre os grupos ecológicos ao longo dos cinco inventários foram avaliadas pelo teste de Qui-quadrado (Tabela de Contingência) (Zar 1999).

\section{Resultados}

Mudanças na riqueza e

na diversidade de espécies

Ao longo dos cinco levantamentos realizados na FVVN foram registrados 1.865 indivíduos distribuídos em 187 espécies, 128 gêneros e 53 famílias (Tab. 1). Desse total, 11 espécies tiveram sua identificação limitada à categoria taxonômica de gênero, três à categoria de família e seis espécies não foram identificadas (Tab. 1). O número de espécies registradas em cada levantamento variou entre 148 e 168 espécies (Tab. 2). A riqueza potencial de espécies arbustivas e arbóreas, obtida pelo estimador Jackknife 1, indicou que o número de espécies registradas em todo período de análise representou entre $74,5 \%$ e $76,2 \%$ da riqueza estimada para a comunidade (Tab. 2). O número de espécies representadas por um único indivíduo variou entre $28,4 \%$ e $30,9 \%$ nos cinco levantamentos realizados (Tab. 2).

Em relação à mudança no número de espécies no intervalo TG, houve aumento líquido de 20 espécies como consequência do surgimento de $34(23,7 \%)$ e do desaparecimento de $14(9,5 \%)$ (Tab. 1) (Tab. 3), o que representou contraste significativo $(Z=2,89 ; p=0,005)$. Das 34 espécies que ingressaram na amostragem, 22 são climáxicas exigentes de luz, sete são climáxicas tolerantes à sombra e cinco não foram identificadas ou não tiveram o grupo ecológico determinado (Tab. 4). Das 14 espécies que não tiveram registro no inventário de 2010, cinco são climáxicas exigentes de luz, cinco são climáxicas tolerantes à sombra e quatro são pioneiras.

Em função do balanço positivo entre entrada e saída de espécies no intervalo TG, foi observada maior riqueza em 2010 em relação à de 1996 (Fig. 2). Considerando-se o mesmo número de indivíduos, para ajuste do esforço amostral, para 
Tabela 1 - Espécies arbustivas e arbóreas amostradas na Floresta de Vale do Véu de Noiva, Parque Nacional da Chapada dos Guimarães, MT. As espécies estão dispostas em ordem alfabética seguidas de suas respectivas famílias botânicas, hábitos, grupos ecológicos (G.E.) e abundâncias registradas nos inventários (1996, 1999, 2003, 2006, 2010), onde: A = árvore, $\mathrm{a}=$ arbusto; $\mathrm{CL}=$ clímax exigente de luz, $\mathrm{CS}=$ clímax tolerante à sombra; $\mathrm{P}=$ pioneira e $\mathrm{NI}=$ não identificada. Table 1 - Tree species sampled in a valley forest, Chapada dos Guimarães National Park, MT. The species are arranged in alphabetical order followed by their botanical famailies, habits, successional groups (G.E.) and abundances recorded in surveys (1996, 1999, 2003, 2006, 2010), where: $\mathrm{A}=$ tree, $\mathrm{a}=$ shrub; $\mathrm{CL}=$ light demanding climax, $\mathrm{CS}=$ shade tolerant climax and $\mathrm{P}=$ pioneer; $\mathrm{NI}=$ unidentified.

\begin{tabular}{|c|c|c|c|c|c|c|c|c|}
\hline Nome científico & Família & Hábito & G.E. & 1996 & 1999 & 2003 & 2006 & 2010 \\
\hline Acacia sp. & Fabaceae & A & $\mathrm{CL}$ & & & & 1 & 2 \\
\hline Aegiphila brachiata Vell. & Lamiaceae & a & $\mathrm{P}$ & 1 & & & & \\
\hline Aegiphila verticillata Vell. & Lamiaceae & a & $\mathrm{P}$ & 1 & & & & \\
\hline Aiouea cf. trinervis Meisn. & Lauraceae & a & CS & & & & & 1 \\
\hline Alchornea glandulosa Poepp. \& Endl. & Euphorbiaceae & A & CL & 8 & 7 & 7 & 6 & 5 \\
\hline Allophylus edulis (A.St.-Hil. et al.) Hieron. ex Niederl. & Sapindaceae & $\mathrm{A}$ & $\mathrm{CL}$ & 5 & 5 & 6 & 6 & 3 \\
\hline Amaioua guianensis Aubl. & Rubiaceae & A & CL & 7 & 7 & 8 & 9 & 11 \\
\hline Antonia ovata Pohl & Loganiaceae & A & CL & 1 & 1 & 1 & 1 & 1 \\
\hline Apeiba tibourbou Aubl. & Malvaceae & A & CL & 1 & 1 & 1 & & \\
\hline Apuleia leiocarpa (Vogel) J.F.Macbr. & Fabaceae & A & $\mathrm{CL}$ & 7 & 7 & 7 & 6 & 6 \\
\hline Aspidosperma spruceanum Benth. ex Müll.Arg. & Apocynaceae & $\mathrm{A}$ & CS & 2 & 1 & 1 & 1 & 1 \\
\hline Astrocaryum aculeatum G.Mey. & Arecaceae & A & $\mathrm{CS}$ & 1 & 1 & 1 & 1 & 1 \\
\hline Astronium fraxinifolium Schott & Anacardiaceae & A & $\mathrm{CL}$ & 1 & 1 & 1 & 1 & 1 \\
\hline Attalea phalerata Mart. ex Spreng. & Arecaceae & A & CS & 2 & 2 & 2 & 2 & 2 \\
\hline Banara tomentosa $\mathrm{Clos}$ & Salicaceae & A & $\mathrm{CL}$ & 1 & & & & \\
\hline Bauhinia mollis (Bong.) D.Dietr. & Fabaceae & a & CL & 4 & 3 & 3 & 4 & 3 \\
\hline Bellucia grossularioides (L.) Triana & Melastomataceae & a & $\mathrm{CS}$ & 5 & 5 & 4 & 3 & 2 \\
\hline Bocageopsis mattogrossensis (R.E.Fr.) R.E.Fr. & Annonaceae & A & CL & 28 & 31 & 32 & 33 & 34 \\
\hline Buchenavia tetraphylla (Aubl.) R.A.Howard & Combretaceae & A & CL & 2 & 2 & 3 & 3 & 2 \\
\hline Byrsonima coriacea (Sw.) DC. & Malpighiaceae & A & CL & 1 & 1 & 1 & 2 & 1 \\
\hline Calyptranthes lucida Mart. ex DC. & Myrtaceae & A & $\mathrm{CS}$ & 47 & 43 & 46 & 46 & 46 \\
\hline Calyptranthes strigipes O.Berg & Myrtaceae & a & $\mathrm{CL}$ & 2 & 2 & 2 & 3 & 2 \\
\hline Casearia arborea (Rich.) Urb. & Salicaceae & A & $\mathrm{CS}$ & 6 & 8 & 8 & 8 & 10 \\
\hline Casearia gossypiosperma Briq. & Salicaceae & A & CL & 2 & 2 & 3 & 1 & 1 \\
\hline Cecropia pachystachya Trécul & Urticaceae & A & $P$ & 14 & 10 & 8 & 3 & 2 \\
\hline Ceiba speciosa (A.St.-Hil.) Ravenna & Malvaceae & A & CL & 1 & 1 & 1 & 1 & 1 \\
\hline Cheiloclinium cognatum (Miers) A.C.Sm. & Celastraceae & A & $\mathrm{CS}$ & 15 & 15 & 16 & 15 & 14 \\
\hline Chloroleucon tortum (Mart.) Pittier & Fabaceae & A & $\mathrm{CL}$ & 1 & 1 & 1 & 1 & 1 \\
\hline Chrysophyllum amazonicum T.D.Penn. & Sapotaceae & A & $\mathrm{CL}$ & 11 & 11 & 12 & 11 & 9 \\
\hline Chrysophyllum gonocarpum (Mart. \& Eichler ex Miq.) Engl. & Sapotaceae & A & CS & 6 & 6 & 6 & 6 & 6 \\
\hline Coccoloba mollis Casar. & Polygonaceae & A & CL & 1 & 1 & 1 & 1 & 1 \\
\hline Connarus perrottetii (DC.) Planch. & Connaraceae & A & CL & 7 & 8 & 7 & 11 & 13 \\
\hline Copaifera langsdorffii Desf. & Fabaceae & A & CS & 5 & 5 & 4 & 4 & 4 \\
\hline Cordia bicolor A.DC. & Boraginaceae & A & $\mathrm{CL}$ & 6 & 6 & 6 & 7 & 6 \\
\hline Cordiera macrophylla (K.Schum.) Kuntze & Rubiaceae & a & CS & 4 & 6 & 6 & 6 & 5 \\
\hline Cordiera sp. & Rubiaceae & A & CL & 9 & 9 & 10 & 10 & 10 \\
\hline Coussarea hydrangeifolia (Benth.) Müll.Arg. & Rubiaceae & a & $\mathrm{CS}$ & 11 & 10 & 10 & 13 & 13 \\
\hline
\end{tabular}




\begin{tabular}{|c|c|c|c|c|c|c|c|c|}
\hline Nome científico & Família & Hábito & G.E. & 1996 & 1999 & 2003 & 2006 & 2010 \\
\hline Cybianthus brasiliensis (Mez) G.Agostini & Primulaceae & a & $\mathrm{CS}$ & 1 & 1 & & 1 & 1 \\
\hline Cybianthus guyanensis (A.DC.) Miq. & Primulaceae & a & $\mathrm{CS}$ & & & & 1 & 1 \\
\hline Diospyros sericea A.DC. & Ebenaceae & A & $\mathrm{CL}$ & & & 1 & 1 & 2 \\
\hline Diptychandra aurantiaca Tul. & Fabaceae & A & $\mathrm{CL}$ & 1 & 1 & 1 & 1 & 1 \\
\hline Duguetia echinophora R.E.Fr. & Annonaceae & A & $\mathrm{CL}$ & 2 & 2 & 2 & 3 & 3 \\
\hline Ecclinusa ramiflora Mart. & Sapotaceae & A & $\mathrm{CS}$ & 56 & 59 & 63 & 61 & 61 \\
\hline Elaeoluma glabrescens (Mart. \& Eichler) Aubrév. & Sapotaceae & A & $\mathrm{CS}$ & 7 & 8 & 8 & 8 & 8 \\
\hline Emmotum nitens (Benth.) Miers & Icacinaceae & $\mathrm{A}$ & $\mathrm{CL}$ & 1 & 1 & 2 & 2 & 4 \\
\hline Endlicheria paniculata (Spreng.) J.F.Macbr. & Lauraceae & A & $\mathrm{CS}$ & 1 & 1 & 1 & & \\
\hline Eriotheca gracilipes (K.Schum.) A.Robyns & Malvaceae & A & $\mathrm{CL}$ & 1 & 1 & 1 & 1 & 1 \\
\hline Erythroxylum anguifugum Mart. & Erythroxylaceae & a & $\mathrm{CS}$ & 1 & 2 & 1 & 1 & 2 \\
\hline Eugenia florida DC. & Myrtaceae & A & $\mathrm{CL}$ & 17 & 19 & 23 & 21 & 20 \\
\hline Eugenia uniflora L. & Myrtaceae & A & $\mathrm{CL}$ & 1 & 1 & & & \\
\hline Ficus guaranitica Chodat & Moraceae & A & $\mathrm{CS}$ & & & 1 & 1 & 1 \\
\hline Ficus insipida Willd. & Moraceae & A & CS & 1 & 1 & 1 & 1 & 1 \\
\hline Ficus krukovii Standl. & Moraceae & A & $\mathrm{CS}$ & 1 & 1 & 1 & & \\
\hline Ficus maxima Mill. & Moraceae & A & $\mathrm{CS}$ & 1 & 1 & 1 & 1 & 1 \\
\hline Guarea guidonia (L.) Sleumer & Meliaceae & A & $\mathrm{CS}$ & 10 & 8 & 7 & 6 & 6 \\
\hline Guarea kunthiana A.Juss. & Meliaceae & A & $\mathrm{CS}$ & 6 & 6 & 6 & 5 & 5 \\
\hline Guatteria nigrescens Mart. & Annonaceae & A & $\mathrm{CS}$ & 18 & 18 & 16 & 16 & 14 \\
\hline Guatteria olivacea R.E.Fr. & Annonaceae & A & $\mathrm{CL}$ & 1 & 1 & 3 & 4 & 4 \\
\hline Guazuma ulmifolia Lam. & Malvaceae & A & CL & 1 & 1 & 1 & 1 & 1 \\
\hline Guettarda sp. & Rubiaceae & a & $\mathrm{CL}$ & & & & & 1 \\
\hline Handroanthus serratifolius (A.H.Gentry) S.Grose & Bignoniaceae & A & $\mathrm{CL}$ & 3 & 3 & 3 & 3 & 3 \\
\hline Heisteria densifrons Engl. & Olacaceae & A & $\mathrm{CL}$ & 2 & 2 & 2 & 2 & 2 \\
\hline Heisteria ovata Benth. & Olacaceae & A & $\mathrm{CL}$ & 6 & 7 & 8 & 8 & 12 \\
\hline Heteropterys byrsonimiifolia A.Juss. & Malpighiaceae & A & $\mathrm{CL}$ & & & 1 & & \\
\hline Himatanthus sucuuba (Spruce ex Müll.Arg.) Woodson & Apocynaceae & A & $\mathrm{CL}$ & 3 & 3 & 5 & 5 & 4 \\
\hline Hirtella glandulosa Spreng. & Chrysobalanaceae & A & $\mathrm{CL}$ & 2 & 2 & 3 & 4 & 5 \\
\hline Hymenaea courbaril L. & Fabaceae & A & $\mathrm{CS}$ & 10 & 12 & 12 & 12 & 11 \\
\hline Ilex sp. & Aquifoliaceae & A & $\mathrm{CL}$ & & & 1 & 1 & 1 \\
\hline Inga cayennensis Sagot ex Benth. & Fabaceae & A & $\mathrm{CL}$ & 5 & 8 & 12 & 10 & 7 \\
\hline Inga cf. cylindrica (Vell.) Mart. & Fabaceae & A & $\mathrm{CL}$ & & & & 1 & 2 \\
\hline Inga edulis Mart. & Fabaceae & A & $\mathrm{CL}$ & & & & 1 & 1 \\
\hline Inga heterophylla Willd. & Fabaceae & A & $\mathrm{CL}$ & & 2 & 7 & 10 & 11 \\
\hline Inga marginata Willd. & Fabaceae & A & CL & 5 & 8 & 10 & 14 & 14 \\
\hline Inga nobilis Willd. & Fabaceae & A & $\mathrm{CL}$ & & & & & 1 \\
\hline Inga vera Willd. & Fabaceae & A & CL & 22 & 23 & 24 & 25 & 22 \\
\hline Iryanthera sp. & Myristicaceae & A & $\mathrm{CS}$ & & & & & 2 \\
\hline Jacaranda copaia (Aubl.) D.Don & Bignoniaceae & A & $\mathrm{CL}$ & 13 & 11 & 10 & 9 & 9 \\
\hline Jacaranda cuspidifolia Mart. & Bignoniaceae & A & CL & & 2 & 1 & 1 & 1 \\
\hline Koanophyllon tinctorium Arruda & Asteraceae & a & $\mathrm{CL}$ & 1 & 1 & 1 & & \\
\hline Lauraceae & Lauraceae & A & $\mathrm{CL}$ & & & & & 1 \\
\hline
\end{tabular}




\begin{tabular}{|c|c|c|c|c|c|c|c|c|}
\hline Nome científico & Família & Hábito & G.E. & 1996 & 1999 & 2003 & 2006 & 2010 \\
\hline Licania apetala (E.Mey.) Fritsch & Chrysobalanaceae & A & $\mathrm{CL}$ & 8 & 7 & 8 & 8 & 8 \\
\hline Licania hoehnei Pilg. & Chrysobalanaceae & $\mathrm{A}$ & $\mathrm{CL}$ & 5 & 4 & 5 & 5 & 3 \\
\hline Licania kunthiana Hook.f. & Chrysobalanaceae & A & CL & 3 & 3 & 4 & 5 & 4 \\
\hline Licania sclerophylla (Hook.f.) Fritsch & Chrysobalanaceae & A & $\mathrm{CL}$ & 4 & 5 & 4 & 4 & 4 \\
\hline Mabea piriri Aubl. & Euphorbiaceae & A & $\mathrm{P}$ & 4 & 4 & 3 & 4 & 2 \\
\hline Machaerium brasiliense Vogel & Fabaceae & A & $\mathrm{CL}$ & 11 & 10 & 9 & 9 & 8 \\
\hline Maclura tinctoria (L.) D.Don ex Steud. & Moraceae & A & $\mathrm{CL}$ & 1 & 1 & 1 & 1 & 1 \\
\hline Manihot tripartita (Spreng.) Müll.Arg. & Euphorbiaceae & a & $\mathrm{P}$ & 1 & 2 & 1 & 1 & \\
\hline Maprounea guianensis Aubl. & Euphorbiaceae & A & $\mathrm{CL}$ & 13 & 14 & 14 & 15 & 23 \\
\hline Matayba elaeagnoides Radlk. & Sapindaceae & A & $\mathrm{CL}$ & 28 & 25 & 26 & 28 & 36 \\
\hline Matayba guianensis Aubl. & Sapindaceae & A & $\mathrm{CL}$ & 17 & 16 & 19 & 19 & 15 \\
\hline Metrodorea stipularis Mart. & Rutaceae & A & $\mathrm{CS}$ & 3 & 3 & 3 & 2 & 3 \\
\hline Miconia albicans (Sw.) Triana & Melastomataceae & a & $\mathrm{P}$ & 11 & 8 & 4 & 6 & 4 \\
\hline Miconia chartacea Triana & Melastomataceae & a & $\mathrm{CS}$ & 19 & 19 & 18 & 20 & 16 \\
\hline Miconia longifolia (Aubl.) DC. & Melastomataceae & a & $\mathrm{CL}$ & 9 & 10 & 9 & 7 & 7 \\
\hline Miconia matthaei Naudin & Melastomataceae & a & $\mathrm{CL}$ & 16 & 17 & 17 & 16 & 16 \\
\hline Miconia minutiflora (Bonpl.) DC. & Melastomataceae & A & $\mathrm{CL}$ & 1 & 1 & 3 & 4 & 4 \\
\hline Miconia nervosa $(\mathrm{Sm}$.$) Triana$ & Melastomataceae & $\mathrm{a}$ & $\mathrm{CS}$ & 13 & 9 & 10 & 10 & 6 \\
\hline Miconia punctata (Desr.) DC. & Melastomataceae & a & $\mathrm{CL}$ & & 1 & 1 & 1 & 1 \\
\hline Miconia sp. 1 & Melastomataceae & a & $\mathrm{CL}$ & & & & 1 & 1 \\
\hline Miconia sp. 2 & Melastomataceae & a & $\mathrm{CL}$ & 5 & 6 & 6 & 5 & 6 \\
\hline Miconia splendens (Sw.) Griseb. & Melastomataceae & A & $\mathrm{CL}$ & & & 1 & 2 & 1 \\
\hline Miconia tomentosa (Rich.) D.Don & Melastomataceae & A & $\mathrm{CS}$ & 1 & 1 & & & \\
\hline Micropholis venulosa (Mart. \& Eichler) Pierre & Sapotaceae & A & CL & 9 & 9 & 10 & 10 & 9 \\
\hline Mollinedia aff. schottiana (Spreng.) Perkins & Monimiaceae & a & $\mathrm{CS}$ & 2 & 2 & 3 & 3 & 3 \\
\hline Mouriri acutiflora Naudin & Melastomataceae & A & $\mathrm{CL}$ & & 1 & 1 & & \\
\hline Myrcia eriocalyx DC. & Myrtaceae & A & $\mathrm{CL}$ & 1 & 1 & 1 & 1 & 1 \\
\hline Myrcia guianensis (Aubl.) DC. & Myrtaceae & A & $\mathrm{CL}$ & 1 & 1 & 1 & 1 & 2 \\
\hline Myrcia sp. & Myrtaceae & A & $\mathrm{CL}$ & & 1 & 1 & 1 & 1 \\
\hline Myrcia splendens (Sw.) DC. & Myrtaceae & A & $\mathrm{CL}$ & 1 & 1 & 1 & 3 & 6 \\
\hline Myrcia tomentosa (Aubl.) DC. & Myrtaceae & A & $\mathrm{CL}$ & 2 & 2 & 2 & 2 & 3 \\
\hline Myrciaria floribunda (H.West ex Willd.) O.Berg & Myrtaceae & A & $\mathrm{CS}$ & 2 & 2 & 2 & 2 & 2 \\
\hline Myrsine lancifolia Mart. & Primulaceae & A & $\mathrm{CL}$ & 3 & 3 & 3 & 4 & 5 \\
\hline Nectandra cuspidata Nees & Lauraceae & A & $\mathrm{CL}$ & 21 & 25 & 31 & 31 & 20 \\
\hline NI 1 & & & & & & & 1 & 1 \\
\hline NI 2 & & & & & & & 1 & \\
\hline NI 3 & & & & & & & 1 & 1 \\
\hline NI 4 & & & & & & & 1 & 1 \\
\hline NI 5 & & & & & & & & 1 \\
\hline NI 6 & & & & & & & & 1 \\
\hline Ocotea aciphylla (Nees \& Mart.) Mez & Lauraceae & A & $\mathrm{CL}$ & 12 & 13 & 15 & 15 & 16 \\
\hline Ocotea corymbosa (Meisn.) Mez & Lauraceae & A & $\mathrm{CL}$ & 2 & 2 & 5 & 6 & 7 \\
\hline Ocotea elegans $\mathrm{Mez}$ & Lauraceae & A & $\mathrm{CL}$ & 9 & 9 & 10 & 9 & 14 \\
\hline
\end{tabular}




\begin{tabular}{|c|c|c|c|c|c|c|c|c|}
\hline Nome científico & Família & Hábito & G.E. & 1996 & 1999 & 2003 & 2006 & 2010 \\
\hline Ocotea pomaderroides (Meisn.) Mez & Lauraceae & A & $\mathrm{CL}$ & 17 & 17 & 17 & 20 & 15 \\
\hline Ocotea spixiana (Nees) Mez & Lauraceae & A & CL & & & & & 1 \\
\hline Oenocarpus distichus Mart. & Arecaceae & A & $\mathrm{CL}$ & 13 & 13 & 10 & 7 & 4 \\
\hline Ormosia arborea (Vell.) Harms & Fabaceae & A & CL & 15 & 17 & 22 & 22 & 24 \\
\hline Palicourea cf. nitidella (Müll.Arg.) Standl. & Rubiaceae & A & $\mathrm{CS}$ & & 1 & & & \\
\hline Parkia sp. & Fabaceae & A & $\mathrm{CL}$ & & & & 1 & 1 \\
\hline Pera heteranthera (Schrank) I.M.Johnst. & Peraceae & A & $\mathrm{CL}$ & 2 & 2 & 2 & 2 & 2 \\
\hline Physocalymma scaberrimum Pohl & Lythraceae & A & CL & 12 & 12 & 12 & 12 & 12 \\
\hline Piper amalago $\mathrm{L}$. & Piperaceae & a & $\mathrm{CS}$ & 2 & 1 & 1 & 1 & \\
\hline Piper arboreum Aubl. & Piperaceae & a & $\mathrm{CS}$ & 5 & 4 & 4 & 5 & 5 \\
\hline Platymiscium floribundum Vogel & Fabaceae & A & $\mathrm{CL}$ & 2 & 2 & 2 & 2 & 2 \\
\hline Poecilanthe parviflora Benth. & Fabaceae & A & CL & 1 & 1 & 1 & 1 & 1 \\
\hline Pouteria gardneri (Mart. \& Miq.) Baehni & Sapotaceae & A & $\mathrm{CS}$ & & & & 1 & 1 \\
\hline Pouteria ramiflora (Mart.) Radlk. & Sapotaceae & A & CL & 11 & 11 & 11 & 11 & 11 \\
\hline Pouteria torta (Mart.) Radlk. & Sapotaceae & A & CL & 1 & 1 & 1 & 1 & 2 \\
\hline Priogymnanthus hasslerianus (Chodat) P.S.Green & Olacaceae & A & $\mathrm{CL}$ & 9 & 9 & 8 & 7 & 7 \\
\hline Protium heptaphyllum (Aubl.) Marchand & Burseraceae & A & $\mathrm{CL}$ & 24 & 24 & 26 & 25 & 26 \\
\hline Protium pilosissimum Engl. & Burseraceae & A & $\mathrm{CL}$ & 39 & 43 & 40 & 43 & 47 \\
\hline Protium spruceanum (Benth.) Engl. & Burseraceae & A & $\mathrm{CS}$ & 6 & 7 & 8 & 7 & 7 \\
\hline Pseudobombax marginatum(A.St.-Hil.) A. Robyns & Malvaceae & A & $\mathrm{CL}$ & & & & 1 & 2 \\
\hline Pseudolmedia laevigata Trécul & Moraceae & A & $\mathrm{CL}$ & 45 & 43 & 42 & 40 & 40 \\
\hline Psychotria carthagenensis Jacq. & Rubiaceae & A & $\mathrm{CS}$ & & & & 1 & 1 \\
\hline Qualea multiflora Mart. & Vochysiaceae & A & $\mathrm{CL}$ & 8 & 9 & 7 & 6 & 4 \\
\hline Quiina rhytidopus Tul. & Quiinaceae & A & CL & 7 & 9 & 7 & 6 & 4 \\
\hline Rhamnidium elaeocarpum Reissek & Rhamnaceae & A & $\mathrm{CL}$ & & 1 & 1 & 1 & 1 \\
\hline Roupala montana Aubl. & Proteaceae & A & CL & 2 & 2 & 2 & 2 & 2 \\
\hline Rubiaceae & Rubiaceae & $\mathrm{a}$ & $\mathrm{CS}$ & & & & & 1 \\
\hline Sacoglottis mattogrossensis Malme & Humiriaceae & A & $\mathrm{CL}$ & 16 & 17 & 23 & 27 & 25 \\
\hline Sapium glandulosum (L.) Morong & Euphorbiaceae & A & $\mathrm{CL}$ & 11 & 9 & 5 & 4 & 4 \\
\hline Schefflera morototoni (Aubl.) Maguire et al. & Araliaceae & A & $\mathrm{CL}$ & 3 & 3 & 3 & 3 & 3 \\
\hline Schefflera vinosa (Cham. \& Schltdl.) Frodin \& Fiaschi & Araliaceae & a & $\mathrm{CS}$ & & 1 & & & \\
\hline Schwartzia adamantium (Cambess.) Bedell ex Gir.-Cañas & Marcgraviaceae & a & CL & & & & & 1 \\
\hline Sebastiania membranifolia Müll.Arg. & Euphorbiaceae & a & $\mathrm{CL}$ & 7 & 7 & 6 & 4 & 3 \\
\hline Senegalia polyphylla (DC.) Britton \& Rose & Fabaceae & A & $\mathrm{CL}$ & 1 & 1 & 1 & 1 & 1 \\
\hline Simarouba amara Aubl. & Simaroubaceae & A & CL & 1 & 1 & 1 & 1 & 1 \\
\hline Siparuna guianensis Aubl. & Siparunaceae & a & $\mathrm{CS}$ & 76 & 83 & 82 & 71 & 63 \\
\hline Sloanea guianensis (Aubl.) Benth. & Elaeocarpaceae & A & $\mathrm{CL}$ & 6 & 7 & 8 & 7 & 7 \\
\hline Sloanea tuerckheimii Donn.Sm. & Elaeocarpaceae & A & $\mathrm{CL}$ & 44 & 44 & 46 & 46 & 43 \\
\hline Sorocea guilleminiana Gaudich. & Moraceae & a & $\mathrm{CS}$ & 3 & 3 & 3 & 3 & 5 \\
\hline Sparattosperma leucanthum (Vell.) K.Schum. & Bignoniaceae & A & $\mathrm{CL}$ & 3 & 3 & 3 & 3 & 2 \\
\hline Spondias mombin L. & Anacardiaceae & A & $\mathrm{CL}$ & 5 & 3 & 3 & 3 & 3 \\
\hline Sterculia apetala (Jacq.) H.Karst. & Malvaceae & A & CL & 2 & 2 & 2 & 2 & 2 \\
\hline Styrax pohlii A.DC. & Styracaceae & A & $\mathrm{CS}$ & 1 & 1 & 1 & 2 & 1 \\
\hline
\end{tabular}




\begin{tabular}{|c|c|c|c|c|c|c|c|c|}
\hline Nome científico & Família & Hábito & G.E. & 1996 & 1999 & 2003 & 2006 & 2010 \\
\hline Tabebuia roseoalba (Ridl.) Sandwith & Bignoniaceae & A & $\mathrm{CL}$ & & & 1 & 1 & 1 \\
\hline Tachigali vulgaris L.G.Silva \& H.C.Lima & Fabaceae & A & $\mathrm{CL}$ & 13 & 15 & 15 & 15 & 12 \\
\hline Talisia subalbens(Mart.) Radlk. & Sapindaceae & A & $\mathrm{CL}$ & & & & & 1 \\
\hline Tapirira guianensis Aubl. & Anacardiaceae & A & $\mathrm{P}$ & 10 & 12 & 10 & 8 & 6 \\
\hline Tapirira obtusa (Benth.) J.D.Mitch. & Anacardiaceae & A & $\mathrm{P}$ & 1 & 1 & 1 & 2 & 2 \\
\hline Terminalia glabrescens Mart. & Combretaceae & A & $\mathrm{CL}$ & 1 & 1 & 1 & 1 & 1 \\
\hline Tetragastris altissima (Aubl.) Swart & Burseraceae & A & $\mathrm{CL}$ & 4 & 5 & 5 & 4 & 4 \\
\hline Tocoyena brasiliensis Mart. & Rubiaceae & a & $\mathrm{CS}$ & 14 & 15 & 14 & 10 & 10 \\
\hline Trichilia clausseni C.DC. & Meliaceae & A & $\mathrm{CS}$ & 35 & 35 & 39 & 40 & 39 \\
\hline Trichilia pallida $\mathrm{Sw}$. & Meliaceae & A & $\mathrm{CS}$ & 1 & 1 & 1 & 1 & \\
\hline Unonopsis guatterioides (A.DC.) R.E.Fr. & Annonaceae & A & $\mathrm{CS}$ & 31 & 29 & 27 & 24 & 25 \\
\hline Urera baccifera (L.) Gaudich. ex Wedd. & Urticaceae & $\mathrm{a}$ & $\mathrm{CL}$ & 5 & 5 & 3 & 2 & 1 \\
\hline Vatairea macrocarpa (Benth.) Ducke & Fabaceae & A & $\mathrm{CL}$ & 3 & 3 & 4 & 5 & 3 \\
\hline Virola albidiflora Ducke & Myristicaceae & A & $\mathrm{CL}$ & 1 & 1 & & & \\
\hline Virola sebifera Aubl. & Myristicaceae & A & $\mathrm{CL}$ & 7 & 7 & 8 & 10 & 9 \\
\hline Vismia guianensis (Aubl.) Choisy & Hypericaceae & A & $\mathrm{P}$ & 1 & 2 & 2 & 1 & \\
\hline Vochysia haenkeana Mart. & Vochysiaceae & A & $\mathrm{CL}$ & 2 & 2 & 2 & 2 & 2 \\
\hline Xylopia aromatica (Lam.) Mart. & Annonaceae & A & $\mathrm{CL}$ & 4 & 6 & 7 & 9 & 11 \\
\hline Xylopia benthamii R.E.Fr. & Annonaceae & A & $\mathrm{CL}$ & & 1 & 1 & 1 & 2 \\
\hline Xylopia emarginata Mart. & Annonaceae & A & $\mathrm{CL}$ & 3 & 3 & 4 & 4 & 4 \\
\hline Ziziphus oblongifolia S. Moore & Rhamnaceae & A & $\mathrm{CL}$ & 1 & 1 & 1 & 1 & 1 \\
\hline
\end{tabular}

Tabela 2 - Características da vegetação arbustiva e arbórea amostrada na Floresta de Vale do Véu de Noiva, Parque Nacional da Chapada dos Guimarães - MT, para os anos inventariados, onde: $\mathrm{S}_{\mathrm{obs}}=$ riqueza observada, Jack $1=$ estimador de riqueza Jackknife de primeira ordem, $\mathrm{S}_{\text {obs }} \%=$ porcentagem da riqueza observada em relação à riqueza estimada, $\mathrm{Sp} .(\mathrm{n}=1)=$ porcentagem do número de espécies amostradas com um indivíduo, H' = índice de diversidade de Shannon, J' = índice de equabilidade de Pielou e $\mathrm{N}=$ número de indivíduos. Table 2 - Tree community characteristics of a valley forest, Chapada dos Guimarães National Park - MT, for surveys, where: $\mathrm{S}_{\mathrm{obs}}=$ observed richness, Jack $1=$ Jackknife estimator of first order, $\mathrm{S}_{\mathrm{obs}} \%=$ percentage of observed richness in relation to estimated richness, $\mathrm{Sp}(\mathrm{n}=1)=$ percentage of the number of species with one individual, $\mathrm{H}^{\prime}=$ Shannon diversity index, $\mathrm{J}^{\prime}=$ Pielou evenness index and $\mathrm{N}=$ number of individuals.

\begin{tabular}{lccccc}
\hline & $\mathbf{1 9 9 6}$ & $\mathbf{1 9 9 9}$ & $\mathbf{2 0 0 3}$ & $\mathbf{2 0 0 6}$ & $\mathbf{2 0 1 0}$ \\
\hline $\mathrm{S}_{\text {obs }}$ & 148 & 154 & 154 & 162 & 168 \\
Jack 1 & 194,3 & 204,1 & 202,2 & 214,9 & 225,6 \\
$\mathrm{~S}_{\text {obs }}(\%)$ & 76,2 & 75,5 & 76,2 & 75,4 & 74,5 \\
Sp. (n = 1) & $28,4 \%$ & $29,2 \%$ & $29,2 \%$ & $30,9 \%$ & $30,4 \%$ \\
H' & 4,3 & 4,31 & 4,32 & 4,35 & 4,37 \\
J' & 0,86 & 0,86 & 0,86 & 0,86 & 0,85 \\
N & 1.201 & 1.233 & 1.274 & 1.276 & 1.251 \\
\hline
\end{tabular}

o inventário de 2010 foi estimada riqueza de 166 $\pm 1,83$ espécies, a qual foi maior do que a riqueza observada em $1996(Z=-13,26 ; p<0,001)$. Assim, a riqueza registrada em 2010 foi maior do que a observada em 1996, independentemente da diferença no número de indivíduos incluídos na amostra.

As alterações florísticas dos intervalos $\mathrm{T} 1$, $\mathrm{T} 2, \mathrm{~T} 3$ e T4 foram inferiores à do intervalo TG. A entrada de espécies nesses intervalos variou de 9 a 14 espécies (3,9 a 9,09\%), enquanto a saída variou de três a seis espécies $(2,03 \%$ a $3,90 \%)$ (Tab. 3$)$ e nenhum dos contrastes foi significativo $(p>0,05)$. $\mathrm{O}$ intervalo $\mathrm{T} 4$, no qual foi registrado ocorrência do fogo, não apresentou, proporcionalmente, perda e ganho de espécies diferente em relação aos intervalos anteriores (Tab. 3).

As mudanças na riqueza de espécies ao longo do período estudado não refletiram em mudanças significativas nos valores do índice de diversidade de espécies, uma vez que não houve diferença na equabilidade da comunidade (Tab. 2). As pequenas mudanças nos índices de diversidade não foram significativas em nenhuma das comparações realizadas (teste $t$ de Hutcheson; $p>0,05$ ). Os perfis de diversidade dos inventários também 
Tabela 3 - Mudanças na riqueza de espécies arbustivas e arbóreas registrada na Floresta de Vale do Véu de Noiva, Parque Nacional da Chapada dos Guimarães - MT, em cada intervalo analisado (T1 =1996-1999; T2 = 1999-2003; T3 = 2003 - 2006; $\mathrm{T} 4=2006-2010$ e $\mathrm{TG}=1996-2010)$.

Table 3 - Tree species turnover in a valley forest, Chapada dos Guimarães National Park, MT, in each period under analysis $(\mathrm{T} 1=1996-1999 ; \mathrm{T} 2=1999-2003 ; \mathrm{T} 3=2003-2006 ; \mathrm{T} 4=$ $2006-2010$ and $\mathrm{TG}=1996-2010)$.

\begin{tabular}{lccccc}
\hline & T1 & T2 & T3 & T4 & TG \\
\hline Riqueza Inicial & 148 & 154 & 154 & 162 & 148 \\
Ganho de espécies & 9 & 6 & 14 & 11 & 34 \\
& $(6,1 \%)$ & $(3,9 \%)$ & $(9,1 \%)$ & $(6,8 \%)$ & $(23,0 \%)$ \\
Perda de espécies & 3 & 6 & 6 & 5 & 14 \\
& $(2,0 \%)$ & $(3,9 \%)$ & $(3,9 \%)$ & $(3,1 \%)$ & $(9,5 \%)$ \\
Riqueza Final & 154 & 154 & 162 & 168 & 168 \\
\hline
\end{tabular}

evidenciaram a ausência de diferenças significativas na diversidade de espécies (Fig. 3) e indicaram que as pequenas diferenças entre a diversidade dos inventários estão mais associadas às discrepâncias na riqueza do que em relação à abundância relativa das espécies (equabilidade).

\section{Mudanças nos grupos ecológicos}

A comunidade manteve o mesmo padrão de distribuição das espécies entre os grupos ecológicos durante o período estudado. A maior representatividade em número de espécies foi do grupo das climáxicas exigentes de luz (CL), enquanto o grupo das pioneiras foi o menos representativo (Tab. 4). A alteração temporal no número de espécies entre os grupos ecológicos foi baixa e não refletiu em alterações significativas $\left(\mathrm{X}^{2}=1,97 ; p=0,98\right)$. No entanto, apesar de pequenas, as mudanças na composição florística indicaram aumento no grupo das climáxicas exigentes de luz e das climáxicas tolerantes à sombra e de redução nas pioneiras (Tab. 4). Esse último grupo não apresentou ingresso de nenhuma espécie nos anos inventariados e foi apenas registrada a saída de quatro espécies (Aegiphila brachiata, Aegiphila verticillata, Vismia guianensi, Manihot tripartita) ao longo dos 14 anos analisados.

\section{Discussão}

A riqueza registrada ao longo do período de estudo na FVVN foi elevada quando comparada com outras florestas ripárias amostradas no Brasil Central, com metodologia semelhante à utilizada no presente estudo (Felfili 1995a,b; Silva-Júnior
Tabela 4 - Mudanças na riqueza de espécies arbustivas e arbóreas registrada na Floresta de Vale do Véu de Noiva, Parque Nacional da Chapada dos Guimarães - MT, em cada intervalo analisado (T1 = 1996-1999; T2 = 1999-2003; T3 $=2003-2006 ; \mathrm{T} 4=2006-2010$ and $\mathrm{TG}=1996-2010)$ por grupos ecológicos, onde: $\mathrm{CL}=$ clímax exigente de luz; $\mathrm{CS}=$ clímax tolerante à sombra; $\mathrm{P}=$ pioneira.

Table 4 - Tree species turnover in a valley forest, Chapada dos Guimarães National Park, MT, in each period under analysis (T1 = 1996-1999; T2 = 1999-2003; T3 = $2003-2006 ; \mathrm{T} 4=2006-2010$ and $\mathrm{TG}=1996-2010$ ) by successional groups, where: $\mathrm{CL}=$ light demanding climax, $\mathrm{CS}=$ shade tolerant climax and $\mathrm{P}=$ pioneer.

\begin{tabular}{lccccc}
\hline & T1 & T2 & T3 & T4 & TG \\
\hline CL & & & & & \\
Riqueza Inicial & 100 & 106 & 109 & 111 & 100 \\
Ganho de espécies & 7 & 5 & 6 & 6 & 22 \\
Perda de espécies & 1 & 2 & 4 & - & 5 \\
Riqueza Final & 106 & 109 & 111 & 117 & 117 \\
Mudança Líquida (\%) & 6.0 & 2.8 & 1.8 & 5.4 & 17.0 \\
& & & & & \\
CS & & & & & \\
Riqueza Inicial & 39 & 41 & 38 & 40 & 39 \\
Ganho de espécies & 2 & 1 & 4 & 3 & 7 \\
Perda de espécies & - & 4 & 2 & 2 & 5 \\
Riqueza Final & 41 & 38 & 40 & 41 & 41 \\
Mudança Líquida (\%) & 5.1 & -7.3 & 5.3 & 2.5 & 5.1 \\
& & & & & \\
P & & & & & \\
Riqueza Inicial & 9 & 7 & 7 & 7 & 9 \\
Ganho de espécies & - & - & - & - & - \\
Perda de espécies & 2 & - & - & 2 & 4 \\
Riqueza Final & 7 & 7 & 7 & 5 & 5 \\
Mudança Líquida (\%) & -22.2 & - & - & -28.6 & -44.4 \\
\hline & & & & &
\end{tabular}

et al. 2001; Felfili \& Silva-Júnior 1992; Bertani et al. 2001; Schiavini et al. 2001; Lopes \& Schiavini 2007; Oliveira \& Felfili 2008; Guimarães et al. 2008; Miguel et al. 2011; Mews et al. 2012). Apesar da elevada riqueza registrada na FVVN, as estimativas do número potencial de espécies calculadas pelos índices Jackknife 1 sugerem riqueza ainda maior, já que as amostragens realizadas representaram em torno de $75 \%$ das riquezas estimadas. Os elevados valores de riqueza estimada estão relacionados à grande quantidade de espécies localmente pouco abundantes $(\mathrm{N} \leq 2)$, as quais chegaram a representar $45 \%$ da comunidade no inventário de 2010. 


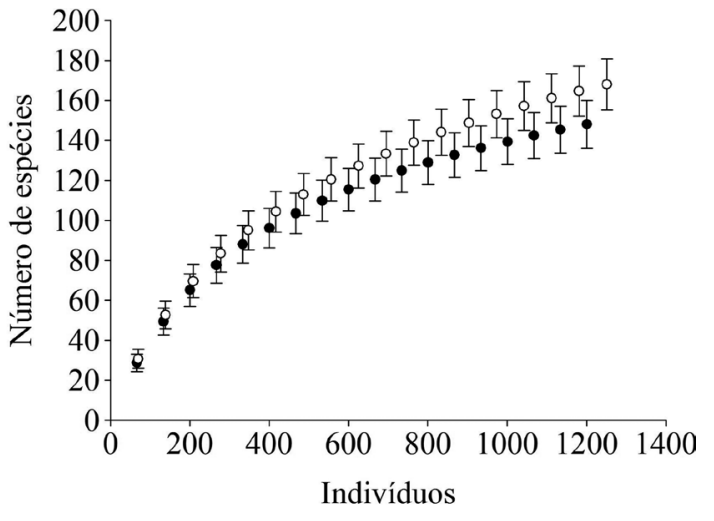

Figura 2 - Curvas de acumulação de espécies arbustivas e arbóreas calculadas por rarefação (Mao Tao) para os inventários realizados em 1996 (•) e 2010 ( ○) na Floresta de Vale do Véu de Noiva, Parque Nacional da Chapada dos Guimarães, MT.

Figure 2 - Accumulation curves of tree species calculated by rarefaction (Mao Tao) for surveys from 1996 (•) and 2010 (०) in a valley forest, Chapada dos Guimarães National Park, MT.

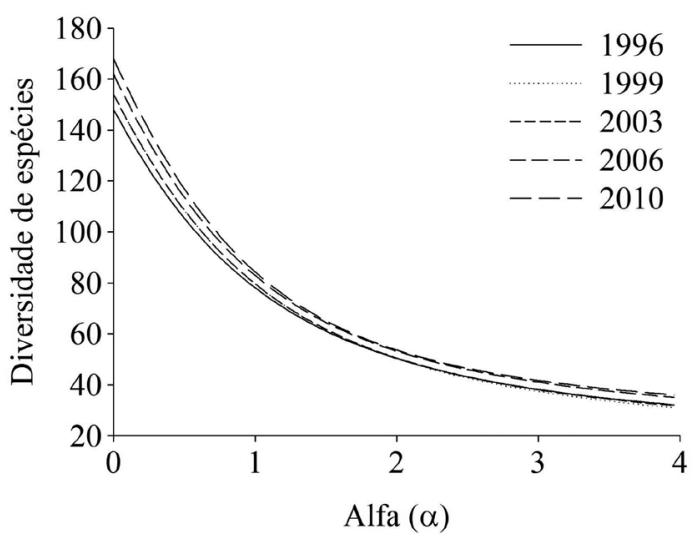

Figura 3 - Perfis de diversidade da vegetação arbustiva e arbórea em cada inventário realizado na Floresta de Vale do Véu de Noiva, Parque Nacional da Chapada dos Guimarães, MT. Figure 3 - Diversity profiles of tree community for surveys in a valley forest, Chapada dos Guimarães National Park, MT.

O elevado número de espécies localmente pouco abundantes registrado em todos os inventários é próximo ao encontrado em outras florestas ripárias brasileiras que empregaram intensidade amostral e critério de inclusão semelhantes (Felfili 1994, 1997; Bertani et al. 2001) e corrobora que aproximadamente um quarto a um terço das espécies nas florestas tropicais são amostradas em baixa densidade (Hartshorn 1980). Além disso, a distribuição de abundâncias das espécies, caracterizada por muitas espécies pouco abundantes e poucas espécies muito abundantes em todos os anos inventariados é citada como característica de florestas tropicais (Felfili \& Felfili 2001; Hartshrorn 1980; Rodrigues \& Leitão-Filho 2000; Silva-Júnior et al. 2001).

Se por um lado a distribuição dos indivíduos entre as espécies observada ao longo do tempo foi semelhante às encontradas em outras formações florestais, as mudanças na riqueza da comunidade parecem diferir das tendências observadas em outros estudos. Comparando os resultados obtidos no intervalo TG com outros trabalhos de dinâmica que analisaram intervalos semelhantes, observa-se maior alteração da riqueza na FVVN. Por exemplo, em uma Floresta Estacional em Minas Gerais Paula et al. (2002) registraram aumento líquido de quatro espécies no período de 14 anos e em uma Mata de Galeria no Distrito Federal Oliveira \& Felfili (2008) observaram ganho de oito espécies em 19 anos de monitoramento. Nesse contexto, os resultados encontrados para TG parecem diferir da teoria de que as florestas tropicais livres de grandes distúrbios ou interferências antrópicas geralmente mudam pouco em relação à composição florística (Swaine et al. 1987). O aumento na riqueza da FVVN no intervalo TG foi superior ao observado em outras florestas analisadas em intervalos semelhantes. Este resultado sugere que a FVVN possui dinâmica florística mais acelerada que outras florestas, embora este padrão possa estar associado ao menor critério mínimo de inclusão adotado no presente estudo (DAP $\geq 5 \mathrm{~cm}$ ) em relação aos demais (DAP $\geq 10 \mathrm{~cm}$ ).

As mudanças nos intervalos menores (T1, T2, T3 e T4), embora também positivas, foram menos perceptíveis em relação às registradas no TG e não apresentaram contrastes significativos, de modo que podem ser consideradas pequenas e semelhantes às mudanças registradas em outros estudos de dinâmica realizadas em florestas tropicais isentas de grandes interferências antrópicas (Oliveria-Filho et al. 1997; Bertani et al. 2001; Werneck et al. 2000; Henriques \& Hay 2002; Mews et al. 2012). Pinto \& Hay (2005) relataram que, de forma geral, o balanço entre entrada e saída de espécies é positivo e o registro de novas espécies em inventários recorrentes numa mesma área varia de 3\% a $10 \%$ e o desaparecimento entre 0 e $8 \%$. Nesse sentido, o balanço positivo entre a saída e entrada de espécies foi observado em todos os intervalos analisados no presente estudo, com exceção do intervalo $\mathrm{T} 2$, no qual o balanço foi nulo. Além disso, os resultados encontrados em relação ao balanço florístico para 
os intervalos T1, T2, T3 e T4 encontram-se dentro dessa faixa de variação descrita para florestas ripárias (Tab. 3). Somente no intervalo TG, o qual apresentou alteração florística significativa, a porcentagem de entrada de espécies extrapolou essa faixa de variação.

Vale destacar que todas as espécies que entraram ou saíram, em qualquer intervalo, foram representadas por um ou dois indivíduos, com exceção de Inga heteroplhylla Willd. na análise do intervalo TG, que entrou na amostragem com 11 indivíduos (Tab. 1). A entrada e saída de espécies concentradas naquelas de baixa densidade corrobora os resultados em outros estudos, nos quais o registro de ocorrência de novas espécies ou o não registro, por ocasião de inventários recorrentes, está relacionado às espécies localmente pouco abundantes (Kellman et al. 1998; Nascimento et al. 1999; Felfili et al. 2000; Werneck et al. 2000; Pinto \& Hay 2005).

É possível que o aumento no número de espécies registradas no intervalo $\mathrm{TG}$ e em todos os intervalos menores esteja relacionado à intensificação de distúrbios naturais na floresta estudada, como relataram Pinto \& Hay (2005). Estes autores indicaram que na FVVN a intensificação das perturbações naturais de pequena intensidade acontece em razão da pouca profundidade do solo, da topografia íngreme e da ação de ventos canalizados que frequentemente incidem no vale. A abertura de clareiras atuaria na modificação das condições ambientais, principalmente luminosidade, temperatura e umidade (Burslem \& Whitmore 1999), o que resultaria em acréscimo de espécies. O fato de a maioria das espécies que entraram em todos os intervalos analisados serem climáxicas exigentes de luz reforça essa sugestão. Assim, a mudança significativa na riqueza da floresta em TG pode ser um indício do alto dinamismo da FVVN.

$\mathrm{O}$ fogo que atingiu umas das vertentes do vale em 2010 parece não ter causado efeitos negativos sobre a riqueza da floresta. $\mathrm{O}$ intervalo T4 (2006-2010), que possibilita avaliação mais específica do efeito do fogo, não apresentou perda mais acentuada de espécies em relação aos intervalos anteriores. A ocorrência do fogo em florestas tropicais geralmente está associada a efeitos negativos sobre a composição florística e estrutura da comunidade, uma vez que esses ecossistemas apresentam poucas adaptações para resistir a esse tipo de distúrbio (Kauffman
\& Uhl 1990; Ivanauskas et al. 2003). Entretanto, respostas aos impactos do fogo variam conforme a intensidade, a frequência e a duração dos incêndios (Hoffmann \& Moreira 2002; Fiedler et al. 2004). Assim, é possível que a intensidade e a duração do fogo ocorrido não tenham sido suficientes para refletir efeitos negativos sobre a riqueza da vegetação arbustiva e arbórea da floresta. Além disso, há possibilidade de os efeitos do fogo serem percebidos e descritos em inventários posteriores, já que indivíduos podem demorar a morrer, como evidenciaram Holdsworth \& Uhl (1998) e Ivanauskas et al. (2003).

Distúrbios à parte, Pinto \& Hay (2005), relataram que o período de três anos (1996-1999) isento de grandes distúrbios parece ter sido curto para tornar significativas as diferenças florísticas e nos índices de diversidade registrado na mesma área do presente estudo. Apesar do intervalo maior (TG) ter apresentado mudanças significativas na composição florística, a manutenção temporal da equabilidade parece ter determinado a ausência de alterações no padrão de diversidade da FVVN no período estudado.

Além da manutenção do padrão de diversidade da floresta ao longo do período de estudo, observou-se estabilidade na contribuição dos grupos ecológicos para a composição florística da vegetação arbórea. A maior representatividade florística das espécies climáxicas exigentes de luz possivelmente é favorecida pela descontinuidade do dossel consequente do relevo acidentado do vale. Além disso, a elevada representatividade desse grupo é citada como característica de florestas ciliares em razão da dinâmica processual deste ecossistema, geralmente caracterizada por frequentes aberturas de clareiras de pequeno porte e pelo efeito de borda na divisa com a vegetação circundante (Felfili 1994; Oliveira-Filho et al. 1994; Felfili 1997; van den Berg \& Oliveira-Filho 1999; Bertani et al. 2001). Paula et al. (2004) comentam que a baixa representatividade florística de espécies pioneiras e de secundárias tardias (análogas às climáxicas tolerantes de sombra, conforme terminologia utilizada neste trabalho) exprime estádio intermediário no tocante ao desenvolvimento sucessional da floresta.

Apesar de pequenas, as alterações florísticas favoráveis às espécies climáxicas exigentes de luz e desfavoráveis ao grupo das pioneiras também apontam estádio intermediário (Werneck et al. 2000; Paula et al. 2004). Entretanto, a intensificação dos 
distúrbios naturais e a inexistência de dossel fechado consequente das características ambientais locais (solo raso, relevo íngreme e correntes de vento no vale) devem ser observadas na classificação do estádio de desenvolvimento desta fitocenose. De acordo com Werneck et al. (2000) e Paula et al. (2004), o desaparecimento de espécies pioneiras caracteriza evolução sucessional e, além disso, a redução da riqueza de espécies pioneiras está associada a fases de recuperação pós-distúrbio (Felfili 1995a,b; Werneck et al. 2000; Werneck \& Francheschinelli 2004).

É possível que a ocorrência do fogo registrado em 2010 provoque alterações no estádio sucessional e na fase de recuperação pós-distúrbio identificados na FVVN no período de 1996 a 2010. A abertura de clareiras observadas em algumas unidades amostrais atingidas pelo fogo provavelmente terá como resposta o aumento no número de espécies do grupo das pioneiras, como indicaram Whitmore (1990), Rezende (1998) e Gurevitch et al. (2009).

Tomados em conjunto, os resultados do presente estudo indicam dinâmica acelerada em termos florísticos no período de 14 anos para a FVVN. No entanto, não foram registradas mudanças na diversidade de espécies e na contribuição dos grupos ecológicos para a composição florística da floresta.

\section{Referências}

Angiosperm Phylogeny Group (APG III). 2009. An update of the Angiosperm Phylogeny Group classification for the orders and families of flowering plants: APG III. Botanical Journal of the Linnean Society 161: 105-121

Appolinário, V.; Oliveira-Filho, A.T. \& Guilherme, F.A.G. 2005. Tree population dynamics in a Brazilian tropical semideciduous forest. Revista Brasileira de Botânica. 28: 347-360.

Aquino, F.G.; Oliveira, M.C.; Schiavini, I. \& Ribeiro, J.F. 1999. Dinâmica de população de Anadenanthera macrocarpa e Acacia glomerosa em mata seca semidecídua na estação ecológica do Panga (Uberlândia-MG). Boletim do Herbário Ezechias Paulo Heringer 4: 90-102.

Baker, T.R.; Swaine, M.D. \& Burslem, D.F.R.P. 2003. Variation in tropical forest growth rates: combined effects of functional group composition and resource availability. Perspectives in Plant Ecology, Evolution and Systematics 6: 21-36.

Bertani, D.F.; Rodrigues, R.R.; Batista, J.L.F. \& Shepherd, G.J. 2001. Análise temporal da heterogeneidade florística e estrutural em uma floresta ribeirinha. Revista Brasileira de Botânica 24: 11-23.
Burslem, D.F.R.P. \& Whitmore,T.C. 1999. Species diversity, susceptibility to disturbance and tree population dynamics in tropical rainforest. Journal of Vegetation Science 10: 767-776.

Carvalho, F.A. \& Felfili, J.M. 2011. Variações temporais na comunidade arbórea de uma floresta decidual sobre afloramentos calcários no Brasil Central: composição, estrutura e diversidade florística. Acta Botanica Brasilica 25: 203-214.

Colwell, R.K. 2006. EstimateS: statistical estimation of species richness and shared species from samples. Version 8. Disponível em <http:purl.oclc.org/ estimates $>$. Acesso em 20 Set 2012.

Colwell, R.K.; Mao, C.X. \& Chang, J. 2004. Interpolatin, extrapolatin, and comparing incidence-based species accumulation curves. Ecology 85: 2717-2727.

Condit, R. 1995. Research in large, long-term tropical forest plots. Tree 10: 18-22.

Connell, J.H. 1978. Diversity in tropical rain forest and reefs. Science 199: 1302-1310.

Eiten, G. 1994. Vegetação do cerrado. In: Pinto, M.N. (ed.) Cerrado: caracterização, ocupação e perpectivas. Ed. UnB, Brasília. Pp. 17-73.

Felfili, J.M. 1994. Floristic composition and phytosociology of gallery forest alongside the Gama stream in Brasília, DF. Brazil. Revista Brasileira de Botânica 17: 1-11.

Felfili, J.M. 1995a. Growth, recruitment and mortality in the Gama gallery forest in central Brazil over a six-year period (1985-1991). Journal of Tropical Ecology 11: 67-83.

Felfili, J.M. 1995b. Diversity, structure and dynamics of a gallery forest in central Brazil. Vegetatio 117: 1-15.

Felfili, J.M. 1997. Dynamics of the natural regeneration in the Gama gallery forest in Central Brazil. Forest Ecology and Management 91: 235-245.

Felfili, M.C. \& Felfili, J.M. 2001. Diversidade alfa e beta no cerrado sensu stricto da Chapada Pratinha, Brasil. Acta Botanica Brasilica 15: 243-254.

Felfili, J.M. \& Silva-Júnior, M.C. 1992. Floristic composition, phytosociology and comparison of cerrado and gallery forests at Fazenda Água Limpa, Federal District, Brazil. In: Furley, P. A. \& Ratter, J.A. (eds.). Nature and dynamics of forest savanna boundaries. Chapman \& Hall, London. Pp. 349-415.

Felfili, J.M.; Rezende, A.V.; Silva-Júnior, M.C. \& Silva, M.A. 2000. Changes in the floristic composition of cerrado sensu stricto in Brazil over a nine-year period. Journal of Tropical Ecology 16:579-590.

Fiedler, N.C.; Azevedo, I.N.C.; Resende, A.V.; Medeiros, M.B. \& Venturoli, F. 2004. Efeito de incêndios florestais na estrutura e composição florística de uma área de cerrado sensu stricto na Fazenda Água Limpa - DF. Revista Árvore 28: 129-138.

Forzza, R.C. et al. (eds.). 2012. Lista de espécies da flora do Brasil. Jardim Botânico do Rio de Janeiro. Disponível em <http://floradobrasil.jbrj.gov. br/2012>. Acesso em 26 Jun 2012. 
Gotelli, N.J. \& Entsminger, G.L. 2001 EcoSim: Null models software for ecology, Version 7.0. Acquired Intelligence Inc. \& Kesey-Bear.

Guimarães, J.C.C.; van den Berg, E.; Castro, G.C.; Machado, E.L.M. \& Oliveira-Filho, A.T. 2008. Dinâmica do componente arbustivo-arbóreo de uma floresta de galeria aluvial no planalto de Poços de Caldas, MG, Brasil. Revista Brasileira de Botânica 31:621-632.

Gurevitch, J.; Scheiner, S.M. \& Fox, G.A. 2009. Ecologia vegetal. 2a ed. Artmed, Porto Alegre. 592p.

Hammer, Ø; Harper, D.A.T. \& Ryan, P.D. 2001. PAST: Paleontological statistical software package for education and data analysis. Palaentologia Electronica 4: 1-9.

Hartshorn, G.S. 1980. Neotropical forests dynamics. Biotropica 12: 23-30.

Henriques, R.P.B. \& Hay, J.D.V. 2002. Patterns and dynamics of plant populations. In: Oliveira, P.S. \& Marquiz, R.J. (eds.). The Cerrados of Brazil: ecology and natural history of a Neotropical Savanna. Columbia University Press, New York. Pp.140-158.

Higuchi, P.; Oliveira-filho, A.T.; Bebber, D.P.; Brown, N.D.; Silva, A.C. \& Machado, E.L.M. 2008. Spatial and temporal patterns of tree community dynamics in a tropical forest fragment along a 19-year period. Plant Ecology 199: 125-135.

Hoffmann, W.A. \& Moreira, A.G. 2002. The role of fire in population dynamics of woody plants. In: Oliveira, P.S. \& Marquis, R.J. (eds.). Cerrados of Brazil. Columbia University Press, New York. Pp. 159-177.

Holdsworth, A.R. \& Uhl, C. 1998. O fogo na floresta explorada e o potencial para a redução de incêndios florestais na Amazônia. Série Amazonia. IMAZON, Belém. 14p.

Ibama. 1994. Plano de ação emergencial: documento e informações básicas do Parque Nacional da Chapada dos Guimarães. Instituto Brasileiro do Meio Ambiente e dos Recursos Naturais Renováveis - Ibama/MT, Cuiabá.

ICMBio. 2009. Plano de Manejo do Parque Nacional da Chapada dos Guimarães. Chapada dos Guimarães - MT. Instituto Chico Mendes de Conservação da Biodiversidade. 250p.

Ivanauskas, N. M.; Monteiro, R. \& Rodrigues, R.R. 2003. Alterations following a fire in a forest community of Alto Rio Xingu (Mato Grosso, Brazil). Forest Ecology and Management 184: 239-250.

Kauffman, J.B. \& Uhl, C. 1990. Interactions of anthropogenic activities, fire, and rain forests in the Amazon Basin. In: Goldammer, J.G. (ed.) Fire in the tropical biota: ecosystem processes and global challenges. Springer, Berlin. Pp. 117-134.

Kellman, M.; Tackaberry, R. \& Rigg, L. 1998. Structure and function in two tropical gallery forest communities: implications for forest conservation in fragmented sytems. Journal of Applied Ecology 35: 195-206.

Laurance, W.F.; Albernaz, A.K.M.; Fearnside, P.M.; Vasconcelos, H.L. \& Ferreira, L.V. 2004. Deforestation in Amazonia. Science 304: 1109.

Laurance, F.W.; Nascimento, H.E.M.; Laurance, S.G.; Andrade, A.; Ribeiro, J.E.L.S.; Giraldo, J.P.; Lovejoy, T.E.; Condit, R.; Chave, J.; Harms, K.E. \& D'Angelo, S. 2006. Rapid decay of tree-community composition in Amazonian forest fragments. PNAS 103: 19010-19014.

Lopes, S.F. \& Schiavini, I. 2007. Dinâmica da comunidade arbórea de mata de galeria da Estação Ecológica do Panga, Minas Gerais, Brasil. Acta Botanica Brasilica 21: 249-261.

Ludwig, J.A. \& J.F. Reynolds. 1988. Statistical ecology: A primer on methods and computing. John Wiley, New York. 337p.

Machado, E.L.M. \& Oliveira-Filho, A.T. 2010. Spatial patterns of tree community dynamics are detectable in a small (4 ha) and disturbed fragment of the Brazilian Atlantic forest. Acta Botanica Brasilica 24: 250-261.

Magurran, A.E. 2004. Measuring biological diversity. Blackwell Science, Oxford. 256p.

Melo, A.S. 2008. O que ganhamos 'confundindo' riqueza de espécies e equabilidade num índice de diversidade? Biota Neotropica 8: 21-27.

Mews, H.A.; Marimon, B.S. \& Ratter, J.A. 2012. Observations on the vegetation of Mato Grosso, Brazil. V. Changes in the woody species diversity of a forest in the cerrado amazonian forest transition zone and notes on the forests of the region. Edinburgh Journal of Botany 69: 239-253.

Miguel, A.; Marimon, B.S.; Oliveira, E.A.; Maracahipes, L. \& Marimon-Junior, B.H. 2011. Dinâmica da comunidade lenhosa de uma floresta de galeria na transição Cerrado-Floresta Amazônica no leste de Mato Grosso, em um período de sete anos (1999 a 2006). Biota Neotropica 11: 1-9.

Nascimento, H.E.M.; Dias, A.S.; Tabanez, A.A.J. \& Viana, V.M. 1999. Estrutura e dinâmica de populações arbóreas de um fragmento de floresta estacional semidecidual na região de Piracicaba, SP. Revista Brasileira de Biologia 59: 329-342.

Oliveira, A.P. \& Felfili, J.M. 2008. Dinâmica da comunidade arbórea de uma mata de galeria do Brasil Central em um período de 19 anos (19852004). Revista Brasileira de Botânica 31: 597-610.

Oliveira-Filho, A.T.; Vilela, A.E.; Gavilanes, M.L. \& Carvalho, D.A. 1994. Effect of soil and topography on the distribution of tree species in a tropical riverine forest in south-eastern Brazil. Journal of Tropical Ecology 10: 483-508.

Oliveira-Filho, A.T.; Mello, J.M. \& Scolforo, J.R.S. 1997. Effects of past disturbance and edges on tree 
community structure and dynamic within a fragment of tropical semideciduous forest in south-eastern Brazil over a five-year period (1987-1992). Plant Ecology 131: 45-66.

Oliveira-Filho, A.T.; Carvalho, W.A.C.; Machado, E.L.M.; Higuchi, P.; Appolinário, V.; Castro, G.C.; Silva, A.C.; Santos, R.M.; Borges, L.F.; Corrêa, B.S. \& Bueno, J.M.A. 2007. Dinâmica da comunidade e populações arbóreas da borda e interior de um remanescente florestal na serra da Mantiqueira, Minas Gerais, em um intervalo de cinco anos (1999-2004). Revista Brasileira de Botânica 30: 149-161.

Paiva, L.V.; Araújo, G.M. \& Pedroni, F. 2007. Structure and dynamics of a woody plant community of a tropical semi-deciduous seasonal forest. Revista Brasileira de Botânica 30: 365-373.

Paula, A.; Silva, A.F.; Souza, A.L. \& Santos, F.A.M. 2002. Alterações florísticas e fitossociológicas da vegetação arbórea numa floresta estacional semidecidual em Viçosa - MG. Revista Árvore 26: 743-749.

Paula, A.; Silva, A.F.; De Marco Júnior, P.; Santos, F.A.M. \& Souza,A.L. 2004. Sucessão ecológica da vegetação arbórea em uma floresta estacional semidecidual, Viçosa/MG. Acta Botanica Brasilica 18: 407-424.

Phillips, O. \& Sheil, D. 1997. Forest turnover, diversity and $\mathrm{CO}_{2}$. Trends in Ecology and Evolution 12: 404.

Phillips, O.L.; van der Heijden, G.; Lewis, S.L.; LópezGonzález, G.; Aragão, L.E.O.C.; Jon Lloyd; Malhi, Y.; Monteagudo, A.; Almeida, S. Dávila, E.A.; Amaral, I.; Andelman, S.; Andrade, A.; Arroyo, L.; Aymard, L.; Baker, T.R.; Blanc, L.; Bonal, D.; Oliveira, A.C.A.; Chao,K.J.; Cardozo, N.D.; Costa, L.; Feldpausch, T.R.; Fisher, J.B.; Fyllas, N.M.; Freitas, M.A.; Galbraith, D.; Gloor, E.; Higuchi, N.; Honorio, E.; Jiménez, E.; Keeling, H.; Killeen, T.J.; Lovett, J.C.; Meir, P.; Mendoza, C.; Morel, A.; Vargas, P.N.; Patiño, S.; Peh, K.S.H.; Cruz, A.P.; Prieto, A.; Quesada, C.A.; Ramírez, F.; Ramírez, H.; Rudas, A.; Salamão, R.; Schwarz, M.; Silva, J.; Silveira, M.; Slik, J.W.F.; Sonké, B.; Thomas, A.S.; Stropp, J.; Tapli, J.R.D.; Vásquez, R. \& Vilanova, E. 2010. Drought-mortality relationships for tropical forests. New Phytologist 187: 631-646.

Pinto, J.R.R. \& Oliveira-Filho, A.T. 1999. Perfil florístico da comunidade arbórea de uma floresta de vale no Parque Nacional da Chapada dos Guimarães, Mato Grosso, Brasil. Revista Brasileira de Botânica 22: 53-67.

Pinto, J.R.R. \& Hay, J.D.V. 2005. Mudanças florísticas e estruturais na comunidade arbórea de uma floresta de vale no Parque Nacional da Chapada dos Guimarães, Mato Grosso, Brasil. Revista Brasileira de Botânica 28: 523-539.

Pinto, J.R.R.; Oliveira-Filho, A.T. \& Hay, J.D.V. 2005. Influence of soil and topography on the composition of a tree community in a central brazilian valley forest. Edinburgh Journal of Botany 62: 69-90.
Rees, M.; Condit, R.; Crawley, M.; Pacala, S. \& Tilman, D. 2001. Long-term studies of vegetation dynamics. Science 293: 650-655.

Rezende, A.V. 1998. Importância das Matas de Galeria: manutenção e recuperação. In: Ribeiro, J.F. (ed.). Cerrado: matas de galeria. Embrapa-CPAC, Planaltina. Pp. 3-16.

Rodrigues, R.R. \& Leitão-Filho, H.F. 2000. Matas ciliares: conservação e recuperação. EDUSP/ FAPESP, São Paulo. 320p.

Schiavini, I.; Resende, J.C.F. \& Aquino, F.G. 2001. Dinâmica de populações de espécies arbóreas em matas de galeria e mata mesófila na margem do Ribeirão Panga, MG. In: Ribeiro, J.F.; Fonseca, C.E.L. \& Souza-Silva, J.C. (eds.) Cerrado: caracterização e recuperação de matas de galeria. Embrapa-CPAC, Planaltina. Pp. 267-299.

Silva-Júnior, M.C.; Felfili, J.M.; Walter, B.M.T.; Nogueira, P.E.; Rezende, A.V.; Morais, R.O. \& Nobrega, M.G.G. 2001. Análise da flora arbórea de Matas de Galeria no Distrito Federal. In: Ribeiro, J.F.; Fonseca, C.E.L. \& Souza-Silva, J.C. (eds.) Cerrado: caracterização e recuperação de matas de galeria. Embrapa-CPAC, Planaltina. Pp. 143-191.

Swaine, M.D. 1990. Population dynamics of tree species in tropical forests. In: Holm-Nielsen, L.B.; Nielsen, I.C. \& Balslev, H. (eds.) Tropical forest: botanical dynamics, speciation and diversity. Academic Press, London. Pp. 101-110.

Swaine, M.D. \& Hall, J.B. 1988. The mosaic theory of forest regeneration and the determination of forest composition in Ghana. Journal of Tropical Ecology 4:253-269.

Swaine, M. D. \& Whitmore, T. C. 1988. On the definition of the ecological species groups in tropical rain forests. Vegetatio 75: 81-86.

Swaine, M.D.; Lieberman, D. \& Putz, F.E. 1987. The dynamics of tree populations in tropical forest: a review. Journal of Tropical Ecology 3: 359-366.

Terborgh, J. 1992. Diversity and the tropical rain Forest. New York: Scientific American Library. 53p.

Tilman, D.; Naeem, S.; Knops, J.; Reich, P.; Siemann, E.; Wedin, D.; Ritchie, M. \& Lawton, J. 1997. Biodiversity and ecosystem properties. Science 278: 1866-1867.

Tóthmérész, B. 1995. Comparison of different methods for diversity ordering. Journal of Science 6: 283-290.

van den Berg, E. \& Oliveira-Filho, A. T. 1999. Spatial partitioning among tree species within an area of tropical montane gallery forest in south-eastern Brazil. Flora 194: 249-266.

Werneck, M.S.; Franceschinelli, E.V. \& Tameirão-Neto, E. 2000. Mudança na florística e estrutura de uma floresta decídua durante um período de quatro anos (1994-1998), na região do triângulo mineiro, MG. Revista Brasileira Botânica 23: 399-411. 
Werneck, M.S. \& Franceschinelli, E.V. 2004. Dynamics of a dry forest fragment after the exclusion of human disturbance in southeastern Brazil. Plant Ecology 174: $337-346$.
Whitmore, T.C. 1990. An introduction to tropical rainforest. Clarenton Press, Oxford. 226p.

Zar, J.H. 1999. Biostatistical analysis. $4^{\text {th }}$ ed. Prentice Hall, New Jersey. 929p. 